\title{
Regularização do classificador de Kernel Density Estimation com funções de Base Radial.
}

\author{
Leonardo Vieira da Costa* Cristiano Leite de Castro** \\ * Programa de Pós-Graduação em Engenharia Elétrica, Universidade \\ Federal de Minas Gerais Brasil (Tel: 31-99619-4092; e-mail: \\ lvieirad@ufmg.br). \\ ** Departamento de Engenharia Elétrica, Universidade Federal de \\ Minas Gerais Brasil (e-mail: crislcastro@ufmg.br)
}

\begin{abstract}
The objective of this article is to propose a method based on the modification of the original Kernel Density Estimation (KDE) model with Radial Base functions. This method uses the K-Means to identify the neurons of the hidden layer, an L1 penalty (LARS) is applied to rank the best neurons, and then the selection function is used to determine the ideal number. In addition, is calculated the Euclidean distance of each neuron to the nearest neuron to find the amplitude of the radial base function. According to experiments in five data sets, the method shows significant advantages and indicates a very interesting generalization performance.

Resumo: O objetivo deste artigo é propor um método baseado na modificação do modelo original de cálculo de Kernel Density Estimation (KDE) com funções de Base Radial. Tal método utiliza-se do K-Means para identificar os neurônios da camada escondida, aplica-se uma penalidade $L 1$ ( $L A R S)$ para ranquear os melhores neurônios e posteriormente faz-se uso da função de seleção para determinar o número ideal. Além disso, calcula-se a distância euclidiana de cada neurônio ao neurônio mais próximo para encontrar a amplitude da função de base radial. De acordo com experimentos em cinco conjuntos de dados, o método mostra vantagens significativas e indica possuir um desempenho de generalização muito interessante.
\end{abstract}

Keywords: Kernel, Density Estimation, Função de Base Radial, LARS, K-Means

Palavras-chaves: Kernel Density Estimation, Função de Base Radial, LARS, K-Means.

\section{INTRODUÇÃO}

Com o objetivo de resolver problemas que possuem dados escassos ou esparsos, um método de estimação não paramétrica de densidade foi criado por Thompson and Tapia (1990). Uma grande vantagem é poder utilizar a estimação não paramétrica para qualquer conjunto de dados. Normalmente é utilizada na modelagem de dados onde não existe informação prévia sobre as distribuições. Esses métodos partem do pressuposto que os dados possuem uma estrutura fixa. Desta forma o problema resume em estimar os parâmetros de média e desvio padrão, que ajustam de melhor forma aos dados observados. Existem algumas situações onde encontramos os dados escassos ou esparsos em que os estimadores calculados estão localizados na região que não correspondem ao local que pode explicar melhor as informações analisadas. Neste tipo de situação é possível estimar uma função de densidade para descrever a função geradora dos dados.

Uma abordagem para resolver esse problema por meio de modelos não paramétricos é conhecida como Kernel Density Estimation (KDE) e explicada por Duda et al. (2001). Esta estimativa é realizada através da superposição de funções de densidade, centradas em cada um dos pontos do conjunto de amostras. Uma vantagem desses estima- dores é possuir apenas um parâmetro a ser ajustado que normalmente está relacionado ao Kernel a ser utilizado, como por exemplo, o parâmetro ' $h$ ' que é o raio da função de base radial. Pérez et al. (2009) propõe o conceito de rede baseado no kernel para classificação supervisionada. Tal conceito estima a densidade das variáveis usando kernels.

Dentre alguns classificadores de Kernel destacamos o classificador Bayes Kernel Density Estimation que será utilizado neste trabalho. Um classificador Bayes Kernel Density Estimation (KDE) é um classificador probabilístico baseado na aplicação de teorema de Bayes com hipóteses de independência entre seus atributos Parzen (1962). Um classificador de Bayes assume que a presença ou ausência de uma característica particular, não está relacionada com a presença ou ausência de qualquer outro elemento. A fórmula geral utilizada pelo classificador de Bayes é dada pela equação 1 .

$$
P\left(C_{j} \mid X\right)=\frac{p\left(X \mid C_{j}\right) P\left(C_{j}\right)}{p(X)}
$$

Desta forma $P\left(C_{j} \mid X\right)$ é o termo definido como probabilidade à posteriori e indica a probabilidade da classe ser $C_{j}$ dado que o padrão $X$ foi determinado. O termo $p\left(X \mid C_{j}\right)$ é uma probabilidade condicional que representa 
a probabilidade de $X$ dado que a classe $C_{j}$ foi apresentada, também conhecida como verossimilhança. O termo $P\left(C_{j}\right)$ é a probabilidade a priori, sendo a informação similar ao conhecimento prévio que se tem sobre os dados em relação à predição de determinada amostra pertencer à classe $C_{j}$, levando em consideração apenas as quantidades de amostras em cada classe. O termo $p(X)$ é definido como evidência e, pode ser visto como um fator que tem como objetivo garantir que a soma das probabilidades à posteriori seja igual a um. A descrição do classificador binário de Bayes tem semelhança a uma rede neural de duas camadas conforme Figura 1.

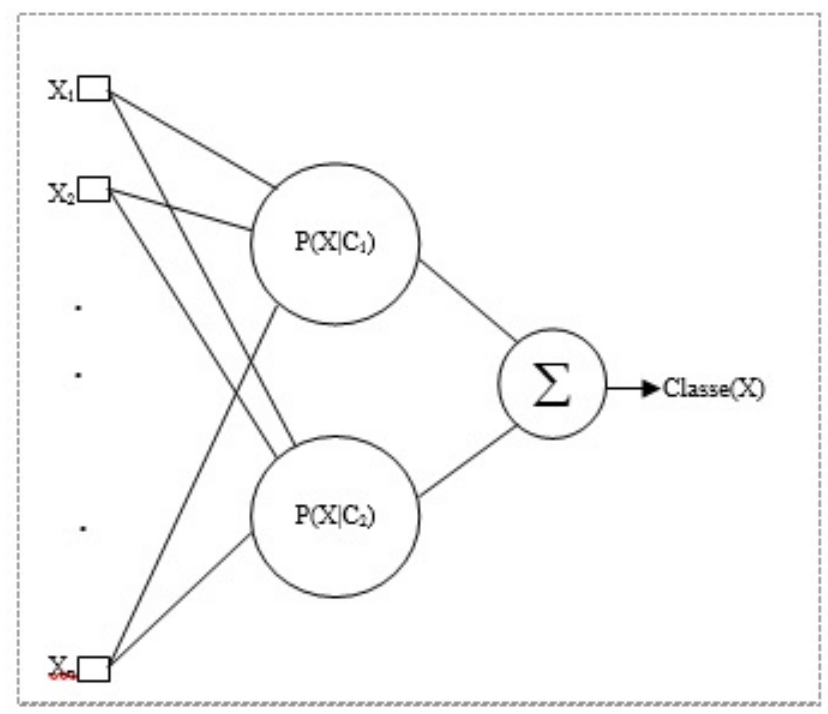

Figura 1. Classificador binário de Bayes na forma de uma rede neural artificial.

\section{METODOLOGIA}

\subsection{Método K-means Cluster}

O método K-means Cluster usa a distância euclidiana entre os pontos para encontrar os centroides. Tal método de análise tem como base o agrupamento não hierárquico por repartição. Isso resulta no procedimento que, dado um número de clusters previamente determinado, calculase pontos que representem os "centros" desses clusters, espalhados de forma homogênea no conjunto de respostas obtidas e movidos, heuristicamente, até grupos preestabelecidos, onde a melhor partição dos 'n' casos será aquela que otimize o critério escolhido. O procedimento é iniciado utilizando os valores das primeiras ' $k$ ' amostras como valores temporários das médias dos clusters. Os centroides iniciais são formados pela qualificação de cada amostra ao cluster de centro mais próximo. Com a inclusão de uma amostra, a média altera-se, modificando assim o centroide.

Um processo iterativo é usado para achar os centroides finais dos clusters. A cada passo, as amostras são agrupadas ao cluster de centro mais próximo, e novamente as médias são recalculadas. Este processo é contínuo e só chega ao final quando não existem mais variações nas médias ou quando atinge um número preestabelecido de iterações Hair et al. (2009). A equação 2 descreve o agrupamento K-means.

$$
E=\sum_{k=1}^{K} \sum_{x_{i} \in C_{k}}^{K} d\left(x_{i} x_{0 k}\right)
$$

Onde $x_{0 k}$ é o centroide do cluster $C_{k}$ e $d\left(x_{i} x_{0 k}\right)$ é a distância euclidiana entre os pontos $x_{j}$ e $x_{0 k}$. Utilizando um critério de otimização que separe uma amostra em ' $k$ ' grupos homogêneos, deseja-se que, dentro de cada grupo, as amostras sejam as mais semelhantes possíveis entre si, ao passo que, as semelhanças entre as amostras de grupos distintos sejam menores possíveis conforme Lourenço et al. (2000).

\subsection{Penalidade $L_{1}$ : LASSO}

Uma parte importante do $K D E$ é minimizar o erro do modelo, que é um problema de regressão comum. A fim de solucionar tal problema pode se aplicar uma técnica denominada $L A S S O$, que utiliza-se de menor encolhimento absoluto de seleção de características proposto por Tibshirani (1996). A solução de Lasso minimiza a soma residual de quadrados, sujeitos à soma do valor absoluto dos coeficientes, que é inferior a uma constante, razão pela qual é também chamado de "penalidade L1". A forma geral em que Lasso trabalha está descrito na equação 3. Devido à natureza da constante, Lasso tende a produzir alguns coeficientes que são zeros e, portanto, produzir modelos interpretáveis. O encolhimento é controlado pelo parâmetro $\lambda$. Quanto menor for o $\lambda$, ocorrerão mais coeficientes de valores zeros e, portanto, menos variáveis serão mantidas no modelo final.

$$
\min _{\lambda, \omega}\left(\sum_{i=1}^{N}\left(y_{i}-x_{j} \omega\right)^{2}+\lambda \sum_{j=1}^{P}\left|\omega_{j}\right|\right)
$$

A regressão de menor ângulo ( $L A R S)$ é uma abordagem mais eficiente do que a $L A S S O$ e foi desenvolvida por Efron et al. (2004). LARS é semelhante à regressão stepwise, mas ao invés de incluir variáveis a cada etapa os parâmetros estimados são incrementados em uma direção equiangular a cada uma das correlações com o residual. Assim, é computacionalmente comparado com a seleção direta. Se duas variáveis possuem um alto grau de correlação com a resposta, então seus coeficientes devem incrementar aproximadamente na mesma taxa. O algoritmo, portanto, é mais estável. Além disso, $L A R S$ é facilmente modificado para produzir soluções para outros estimadores, como o Lasso, e é eficaz quando o número de dimensões é significativamente maior do que o número de amostras conforme Efron et al. (2004). A desvantagem do método $L A R S$ é que ele tem problema com variáveis altamente correlacionadas, mesmo que isso não seja um problema exclusivo desse método.

\subsection{Função de Seleção do Número de Neurônios.}

Uma função de seleção do número de neurônios faz-se necessária após os mesmos terem sidos ranqueados pela técnica do LARS. Existem várias técnicas para resolver esse problema, tais como a de amostra aleatória simples, amostra estratificada, amostra sistemática, amostra por conglomerados, amostra por conveniência, amostra por 
quota, amostra por bola de neve, entre outras. Neste artigo a função proposta para resolução do problema é a raiz quadrada do número de amostras conforme equação 4 . Tal função é normalmente utilizada pelo algoritmo $K$ Nearest Neighbor (KNN) classification que define o número ' $k$ ' de vizinhos a serem avaliados, a fim de resolver o problema de classificação conforme Nagar et al. (2015).

$$
f=\sqrt{n}
$$

\subsection{Raio da função de base radial.}

A função de base radial do tipo Gaussiana é a mais comum a se utilizada em aplicações práticas, e será a função adotada no $K D E$ proposto neste artigo. Tal função consiste no parâmetro ' $h$ ' que define a distância euclidiana média para medir o espalhamento dos dados representados pela função de base radial em torno de seu centro. Os raios de cada uma das funções de base radial de um mesmo $K D E$ podem assumir diferentes valores. A proposta é utilizar diferentes distâncias euclidianas para cada Gaussiana. O cálculo leva em consideração a menor distância euclidiana entre o neurônio selecionado e todos os outros neurônios da camada escondida.

\subsection{Toda a Metodologia}

Para melhor exemplificar a metodologia proposta em problema de classificação, por exemplo, aplica-se diversas etapas para chegar ao resultado esperado, conforme listado nos itens abaixo:

- Primeiramente aplica-se o método K-means para definir quem serão as possíveis candidatas a Gaussiana no modelo final.

- Posteriormente aplica-se a técnica do LARS para ranquear todos os neurônios em ordem de importância.

- Após realizado o ranqueamento feito pelo LARS aplica-se a função de seleção de neurônios, que neste artigo utiliza a raiz quadrada do número de amostras.

- Ao obter-se os neurônios escolhidos calcula-se o raio de cada Gaussiana, que neste caso utiliza a menor distância euclidiana entre os neurônios.

- Por fim gera-se o modelo esperado.

\section{RESULTADOS E DISCUSSÃO}

A fim de testar o método proposto para o problema de classificação, cinco bases de dados encontradas em Dua and Graff (2007) foram escolhidas neste trabalho. As bases de dados serão descritas para melhor exemplificar o desempenho do método proposto frente a Máquinas de Vetores de Suporte (SVM) com Kernel Gaussiano. Toda a implementação foi realizada utilizando o software $R$ Team (2013). Tal software dispõe de alguns algoritmos já implementados como o K-Means e o LARS. Utilizou-se também o pacote 'e1071' que disponibiliza o algoritmo Support Vector Machine.

\subsection{Base de dados Default}

A base de dados Default é um conjunto de registros dos clientes para uma empresa de cartão de crédito. Ela possui 4 características organizadas em 10.000 amostras e apresentado na Tabela 1.
A variável escolhida como resposta foi a Student. Apesar do grande número de amostras a base de dados apresenta uma distribuição bem definida conforme Fig. 2. Por esse motivo os dois métodos obtiveram ótimos desempenhos, tanto na base de treinamento como na base de teste.

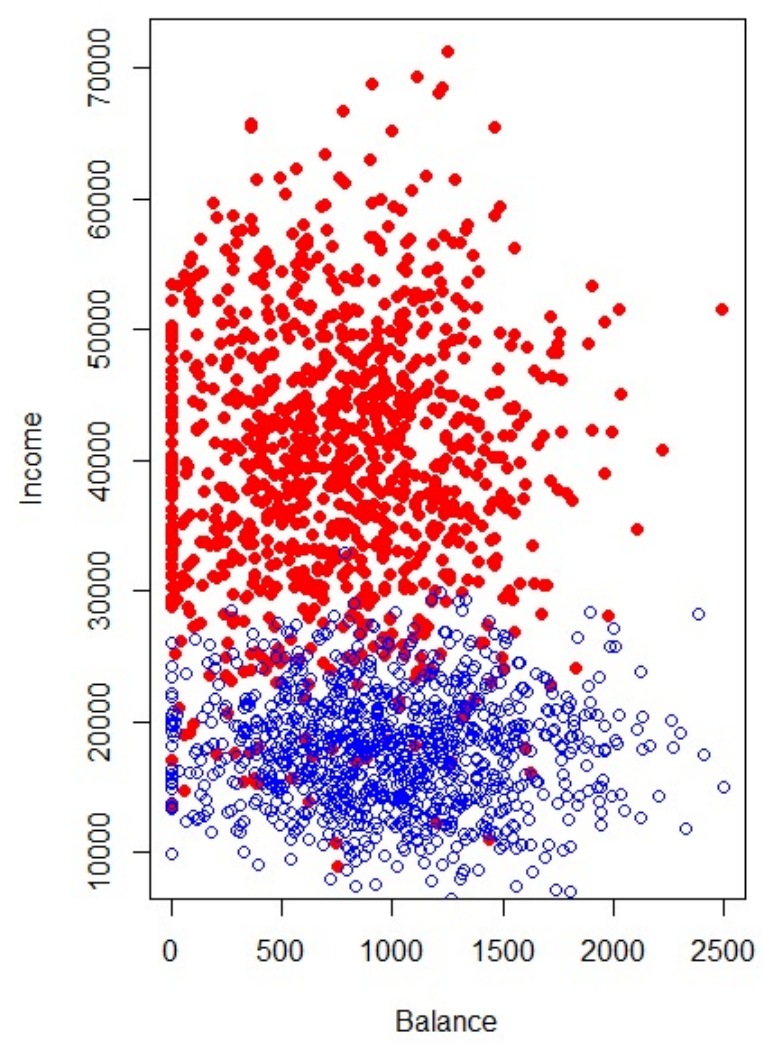

Figura 2. Distribuição dos Dados da base de dados Default.

Tabela 1. Informações sobre a base de dados Default.

\begin{tabular}{cc} 
Atributo & Descrição \\
\hline Default & Yes ou No \\
Student & Yes ou No \\
Income & Contínuo entre 0 e 2654.323 \\
Balance & Contínuo entre 771.9677 e 73554.23 \\
\hline
\end{tabular}

\subsection{Base de dados Iris}

A base de dados Iris tem como objetivo classificar a espécie de planta Iris. O atributo Species possui três classes: Iris Setosa, Iris Versicolour, Iris Virginica. A Base é composta por 5 características e 150 amostras apresentado na Tabela 2 .

Tabela 2. Informações sobre o Bando de Dados Iris.

\begin{tabular}{cc} 
Atributo & Descrição \\
\hline Species & Setosa, Versicolour e Virginica \\
sepal length & Contínuo entre 4.3 e 7.9 \\
sepal width & Contínuo entre 2 e 4.4 \\
petal length & Contínuo entre 1 e 6.9 \\
spetal width & Contínuo entre 0.1 e 2.5 \\
\hline
\end{tabular}

Devido à simplicidade da base e à clara diferenciação entre as classes, o método proposto e o SVM foram capazes de 
classificar corretamente todas as amostras tanto na base de treinamento quanto na base de teste.

\subsection{Base de dados 1985 Auto Imports.}

O conjunto de dados 1985 Auto Imports consiste de 193 amostras. Para cada amostra, 26 atributos podem ser observados como descrito na Tabela 3.

Tabela 3. Informações sobre a base de dados 1985 Auto Imports.

\begin{tabular}{cc} 
Atributo & Descrição \\
\hline symboling & $-3,-2,-1,0,1,2,3$. \\
normalized-losses & Contínuo entre 65 e 256. \\
fuel-type & diesel, gas. \\
aspiration & std, turbo \\
num-of-doors & four, two \\
body-style & hardtop, wagon, sedan, hatchback \\
drive-wheels & 4wd, fwd, rwd. \\
engine-location: & front, rear \\
wheel-base & Contínuo entre 86.6 e 120.9. \\
length & Contínuo entre 141.1 e 208.1 \\
width & Contínuo entre 60.3 e 72.3 \\
height & Contínuo entre 47.8 e 59.8 \\
curb-weight & Contínuo entre 1488 e 4066 \\
engine-type & dohc, dohcv, l, ohc, ohcf, ohcv, rotor \\
num-of-cylinders & eight, five, four, six, three, twelve, two \\
engine-size & Contínuo entre 61 e 326 \\
fuel-system & 1bbl, 2bbl, 4 bbl, idi, mfi, mpfi, spdi, spfi \\
bore & Contínuo entre 2.54 e 3.94 \\
stroke & Contínuo entre 2.07 e 4.17 \\
compression-ratio & Contínuo entre 7 e 23 \\
horsepower & Contínuo entre 48 e 288 \\
peak-rpm & Contínuo entre 4150 e 6600 \\
city-mpg & Contínuo entre 13 e 49 \\
highway-mpg & Contínuo entre 16 e 54 \\
price & Contínuo entre 5118 e 45400 \\
\hline &
\end{tabular}

Uma nova característica foi criada para definir a classificação das amostras baseada na característica existente symboling. Tal característica foi dividida em duas classes, a classe de clientes com baixo risco de análise do seguro que tem valores de symboling menores ou iguais a zero, e a classe com alto risco de análise do seguro que tem valores de symboling maiores do que zero. O método proposto obteve um desempenho um pouco inferior ao SVM na base de treinamento, no entanto, conseguiu um desempenho muito semelhante ao SVM na base de teste.

\subsection{Base de dados Scale Weight \& Distance}

A base de dados Scale Weight \& Distance tem como objetivo modelar resultados experimentais psicológicos. O atributo Class Name possui três classes: left, right $e$ balanced. Neste trabalho reduzimos as amostras para duas classes: left e right. A base é composta por 5 características e 625 amostras, conforme apresentado em Tabela 4 .

Devido à simplicidade da base de dados o método SVM foi capaz de classificar corretamente todas as amostras nas bases de treinamento e teste. O método proposto obteve um alto índice de acerto na base de treinamento e conseguiu classificar corretamente todas as amostras na base de teste.
Tabela 4. Informações sobre a base de Dados Scale Weight \& Distance

\begin{tabular}{cl} 
Atributo & \multicolumn{1}{c}{ Descrição } \\
\cline { 2 - 3 } Class Name & left, right e balanced \\
Left-Weight & Contínuo entre 1 e 5 \\
Left-Distance & Contínuo entre 1 e 5 \\
Right-Weight & Contínuo entre 1 e 5 \\
Right-Distance & Contínuo entre 1 e 5 \\
\hline
\end{tabular}

\subsection{Base de dados House Boston}

A base de dados House Boston tem como objetivo mostrar os valores das casas do subúrbio de Boston. Ele possui 13 características de valores contínuos com 5066 amostras apresentado em Tabela 5.

Tabela 5. Informações sobre a base de dados House Boston.

\begin{tabular}{ll} 
Atributo & Descrição \\
\hline CRIM & $\begin{array}{l}\text { Taxa de criminalidade per capita por ci- } \\
\text { dade } \\
\text { PNoporção de terrenos residenciais por zo- } \\
\text { nas para lotes } 25.000 \text { sq.ft. }\end{array}$ \\
INDUS & $\begin{array}{l}\text { Proporção de negócios não-varejo por ci- } \\
\text { dade }\end{array}$ \\
CHAS & $\begin{array}{l}\text { Lado da cidade em relação ao Rio Charles } \\
\text { Concentração de óxidos nítricos (partes }\end{array}$ \\
NOX & $\begin{array}{l}\text { por } 10 \text { milhões) } \\
\text { Número médio de quartos por habitação }\end{array}$ \\
AGE & $\begin{array}{l}\text { Proporção de IDADE das unidades ocupa- } \\
\text { das pelo proprietário construídas antes de }\end{array}$ \\
DIS & $\begin{array}{l}1940 \\
\text { Distâncias ponderadas a cinco centros de } \\
\text { emprego de Boston }\end{array}$ \\
RAD & $\begin{array}{l}\text { Índice RAD de acessibilidade a estradas } \\
\text { principais }\end{array}$ \\
TAX & $\begin{array}{l}\text { Imposto sobre o Valor Agregado por } \$ \\
10.000\end{array}$ \\
PTRATIO & $\begin{array}{l}\text { Relação aluno-professor por cidade } \\
1000(B k-0,63) \\
\text { de negros por cidade } \\
\text { B status da população com menor renda }\end{array}$ \\
LSTAT & $\begin{array}{l}\text { Valor médio das casas ocupadas por pro- } \\
\text { prietários em } \$ 1000\end{array}$ \\
MEDV &
\end{tabular}

Como o objetivo deste trabalho é calcular a acurácia do modelo proposto, foi criada uma nova característica que leva em conta o valor médio das casas ocupadas por proprietários em \$1000 (MEDV). Foram consideradas casas com valores menores ou iguais a 21.8 como casas da classe "inferiores", e casas acima dos valores de 21.8 como casas da classe "superiores". Depois dessa operação a base ficou balanceada entre as duas classes e posteriormente foram aplicados os dois métodos para verificar a acurácia. O método proposto teve um desempenho menor na base de treinamento. Não obstante obteve uma acurácia muito superior na base de teste.

\subsection{Comparação dos métodos nas cinco bases de dados}

A Fig. 4 mostra a comparação do método proposto neste artigo com o SVM nas cinco bases de dados. Os dados foram divididos entre treinamento e teste, e posteriormente computou-se o percentual da acurácia de cada método.

O método proposto possui uma acurácia muito semelhante à do método SVM em quatro bancos de dados, tanto na 


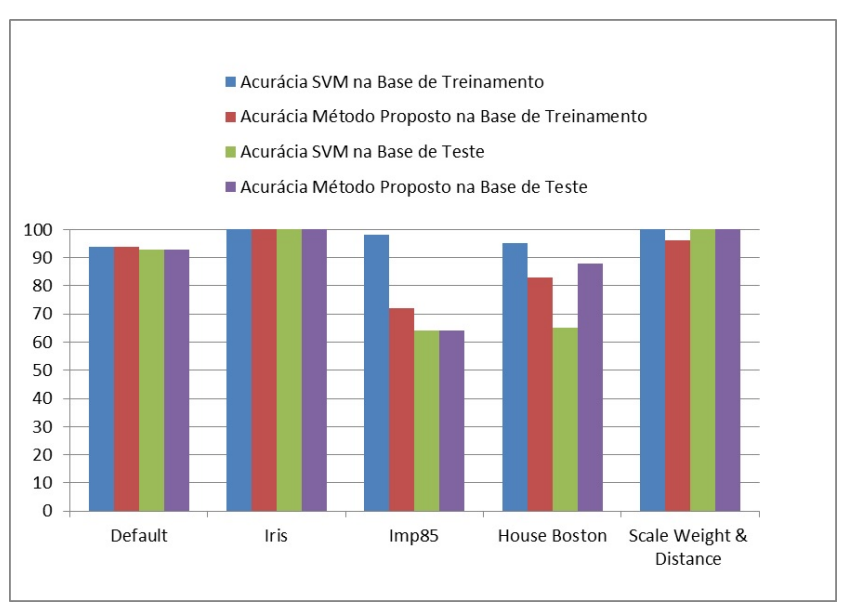

Figura 3. Gráfico com a acurácia dos modelos em \%.

base de treinamento quanto na base teste. É interessante enfatizar que para a base House Boston foi detectado uma melhor acurácia do método proposto em relação ao SVM na base de dados de teste.

\section{CONCLUSÃO}

Este artigo propõe um método que é uma modificação do modelo original de cálculo de Kernel Density Estimation (KDE) com funções de Base Radial.

Tal método utiliza uma penalidade $L 1$ ( $L A R S$ ) para ranquear os melhores neurônios e posteriormente, lança mão de uma função de seleção para determinar o número ideal. Calcula-se a distância euclidiana de cada neurônio ao neurônio mais próximo, para encontrar a amplitude das funções de base radial.

De acordo com os experimentos dos cinco conjuntos de dados, destaca-se a base de dados House Boston, onde o método proposto demostra uma acurácia do conjunto de teste melhor do que o SVM. O método mostra vantagens significativas e indica possuir um desempenho de generalização muito interessante.

Trabalhos futuros sobre este método irão enriquecê-lo, como por exemplo, a modificação da função de seleção de neurônios com uma metodologia estatística, depois de ranqueados pelo $L A R S$.

\section{REFERÊNCIAS}

Dua, D. and Graff, C. (2007). Machine learning repository - uci. University of California, Irvine, School of Information and Computer Sciences, URL http://archive.ics.uci.edu/ml.

Duda, O.Richard, Hart, and Peter, R. (2001). Dg stork pattern classification. John Wiely and Sons.

Efron, B., Hastie, T., Johnstone, I., and Tibshirani, R. (2004). Regression shrinkage and selection via the lasso. Least angle regression, Ann. tatist. 32 (2) (2004), 407-499.

Hair, F.Joseph, Black, William, C., Babin, Barry, J., Anderson, Rolph, Tatham, and Ronald, L. (2009). Análise multivariada de dados. Bookman Editora.

Lourenço, Alexandre, Matias, and P., R. (2000). Estatística multivariada. Instituto Superior de Engenharia do Porto.
Nagar, A., Mohapatra, D.P., and Chaki, N. (2015). Proceedings of 3rd international conference on advanced computing, networking and informatics. ICACNI 2015, 1.

Parzen, E. (1962). On estimation of a probability density function and mode. Ann. Math. Statist, 33(3):10651076.

Pérez, A., Larrañaga, P., and Inza, I. (2009). Bayesian classifiers based on kernel density estimation: Flexible classifiers. International Journal of Approximate Reasoning, 50(2), 341-362.

Team, R.C. (2013). R: A language and environment for statistical computing. $R$ Foundation for Statistical Computing, Vienna, Austria, URL http://www.Rproject.org/.

Thompson, J.R. and Tapia, R.A. (1990). Nonparametric function estimation, modeling, and simulation. Society for Industrial and Applied Mathematics, 21.

Tibshirani, R. (1996). Regression shrinkage and selection via the lasso. J. R. tatist, Soc. 58 (1) (1996), 267-288. 